\title{
The effects of intravitreal adalimumab injection on pseudophakic macular edema
}

\author{
Mohsen Farvardin, Ehsan Namvar*, Fatemeh Sanie-Jahromi and Mohammad Karim Johari
}

\begin{abstract}
Objective: Pseudophakic macular edema is a frequent complication following cataract surgery. Inflammation is a major etiologic factor in the development of pseudophakic cystoid macular edema. Tumor necrosis factor-alpha has an important role in ocular inflammation. Adalimumab (Humira) is an inhibitor of tumor necrosis factor-alpha that has been approved in the United States. An open-label, uncontrolled, prospective, interventional study of five consecutive patients ( 5 eyes) with cystoid macular edema who were treated with off-label intravitreal adalimumab at Khalili Hospital was conducted. Slit-lamp examination and optical coherence tomography were done for all patients.

Results: No statistically significant difference was detected between best corrected visual acuity and central macular thickness before and after injection in pseudophakic macular edema. One patient developed uveitis approximately 2 weeks after injection. Based on the results, adalimumab does not appear to be an effective treatment for pseudophakic macular edema, and it may cause uveitis. Caution should be exercised when using this drug.

Trial registration Iranian Registry of Clinical Trials IRCT2016100430130N1, 2016.12.03, Retrospectively registered
\end{abstract}

Keywords: Pseudophakic macular edema, Adalimumab, Intravitreal injection

\section{Introduction}

pseudophakic macular edema is one of the frequent complications of cataract surgery which is a severe postoperative inflammatory response [1, 2]. Inflammation is generally accepted as the main etiologic factor in the development of pseudophakic cystoid macular edema (CME). It has been shown that postsurgical inflammation may increase the permeability of the blood-retinal barrier (BRB) after cataract surgery, resulting in macular edema $[1,3]$. CME if left untreated can lead to vision loss [4] and so different therapeutic strategies have been so far introduced for CME. Treatments for CME comprise topical, periocular, intravitreal and systemic corticosteroids $[1,5$, 6], systemic and topical nonsteroidal anti-inflammatory drugs (NSAIDs) [1,7], systemic and topical carbonic

*Correspondence: namvar.e@gmail.com

Poostchi Ophthalmology Research Center, Department of Poostchi

Ophthalmology, Shiraz University of Medical Sciences, Zand Street, Shiraz,

Fars, Iran anhydrase inhibitors [1], laser therapy [8], pars plana vitrectomy [9], and intravitreal anti-vascular endothelial growth factor (VEGF) agents [10,11]. Some patients, however, do not respond to the treatments, which represents an unmet need for alternative medicines targeting CME. Tumor necrosis factor-alpha (TNF- $\alpha$ ) is a cytokine released from macrophages/monocytes and is known to have an important role in ocular inflammation [12-14]. Recent reports have confirmed the efficiency of TNF- $\alpha$ inhibition in reducing ocular inflammations [14-16]. Adalimumab (Humira) is the newest antibody against TNF- $\alpha$ which has been approved in the United States as an anti-rheumatic drug [17]. It is a human-derived monoclonal antibody and seems to be much safer than its similar drug -infliximab- which is a mouse-human antibody [18].

To the best of the researchers' knowledge, few studies have been conducted to determine the effects of adalimumab on pseudophakic macular edema; thus, this study was undertaken. 


\section{Main text \\ Methods}

This prospective, interventional study evaluated five consecutive patients (5 eyes) with CME after uncomplicated cataract surgery with no retinal abnormality (such as vitreomacular traction or diabetic abnormality) who were treated with off-label intravitreal adalimumab at Khalili Hospital. The sample size was determined according to previous studies [15-17]. This study was approved by the ethics committee of Shiraz University of Medical Sciences (IR.SUS.REC.1394.99) and Iranian Registry of Clinical Trials (IRCT2016100430130N1) and patient informed written consent were obtained. The drug and its potential risks were described for all patients. This study adheres to CONSORT guidelines and includes a completed CONSORT checklist as an additional file submitted to the journal. Clinical examination included best corrected visual acuity (BCVA), applanation tonometry, slit-lamp examination, fundus examination, and optical coherence tomography (OCT) and was performed on all patients. Baseline retinal thickness was obtained by OCT. A vial of adalimumab $(40 \mathrm{mg} / 0.8 \mathrm{ml})$ was mixed with $0.8 \mathrm{ml}$ of balanced salt solution. Then, $0.04 \mathrm{ml}$ (1 mgofadalimumab) of this solution was prepared for each patient. Each eye was prepared with povodine-iodine $5 \%$, the eyelid speculum was inserted, and $1 \mathrm{mg}(0.04 \mathrm{ml})$ of adalimumab was injected at $3.5 \mathrm{~mm}$ posterior to the limbus with a 30-gauge needle under topical anesthesia. Afterward, a topical antibiotic was administered for 3 days. Patients were given detailed post-injection instructions and asked to refer to the hospital if any pain or decrease in vision occurred.

Case no. 1 received topical NSAID and topical steroid 2 weeks after uncomplicated cataract surgery for 2 months. Then, adalimumab was injected intravitreally. Case no. 2 received 1.5 months of topical NSAID and a topical steroid after uncomplicated cataract surgery. Thereafter, adalimumab was injected intravitreally. Cases no. 3 and 4 received subtenon triamcinolone after one month of using topical NSAID and steroids.
Then, adalimumab was injected intravitreally. Case no. 5 was prescribed topical NSAID and steroids for 1 month followed by subtenon steroid injection. 2 months after uncomplicated cataract surgery, the intravitreal steroid was injected. 3 months later, adalimumab was injected intravitreally.

Slit-lamp biomicroscope and OCT was done for all patients before and after injection. Statistical analysis was performed. The patients' BCVAs were converted to a logarithm of the minimal angle of resolution (logMAR) scale for analysis. A $p$ value $<0.05$ was considered to be significant.

\section{Results}

Five eyes from 5 patients ( 3 men and 2 women) were included in this study. The mean age of the patients was 64.2 years (range $=47-74$ years). Mean logarithms of the minimum angle of resolution of BCVA 1 day before, 1 week after, and 4 weeks after injection were 0.9000 , 0.8380 , and 0.9950 , respectively.

The logarithm of the minimum angle of resolution ranged from 0.52 to 1.77 . The median logarithms of the minimum angle of resolution before, 1 week after, and 4 weeks after injection were $0.6900,0.6900$, and 0.8450 , respectively. (Table 1 ).

There were no statistically significant differences among BCVA values before, 1 week after, and 4 weeks after injection $(p$ value $=0.667,>0.05)$.

There were no statistically significant differences among central macular thickness (CMT) values before, 1 week after, and 4 weeks after injection ( $p$ value $=0.653,>0.05)$.

Case 5 developed uveitis approximately 2 weeks after injection. She presented with red eye and ocular pain. Her intraocular pressure (IOP) had risen. Slit-lamp examination showed conjunctival hyperemia, anterior chamber reaction, multiple keratic precipitates, and vitritis. Anterior chamber and vitreous sampling and intravitreal antibiotic injection were done. Smear and culture were negative, but the patient's condition worsened and visual acuity decreased. Eventually, because of

Table 1 Demographic characteristics, CMT, and BCVA of study patients

\begin{tabular}{|c|c|c|c|c|c|c|c|c|}
\hline Patient No & Eye & Sex & BCVA1 & BCVA2 & BCVA3 & CMT1 & CMT2 & CMT3 \\
\hline 1 & OD & Female & 0.52 & 0.52 & 0.52 & 464 & 505 & 356 \\
\hline 2 & OD & Female & 0.52 & 0.52 & 0.69 & 567 & 371 & $492(422)$ \\
\hline 3 & OS & Male & 1.77 & 1.77 & 1.77 & 968 & 410 & 394 \\
\hline 4 & OD & Male & 1.00 & 0.69 & 1.00 & 406 & 385 (413) & 423 \\
\hline 5 & OD & Female & 0.69 & 0.69 & - & 512 & 571 & - \\
\hline
\end{tabular}

$B C V A 1$ the logarithm of the minimum angle of resolution BCVA 1 day before injection, $B C V A 2$ the logarithm of the minimum angle of resolution $B C V A 1$ week after injection; BCVA3 the logarithm of the minimum angle of resolution BCVA 1 month after injection, CMT1 central macular thickness 1 day before injection, CMT2 central macular thickness 1 week after injection, CMT3 central macular thickness 1 month after injection, OD right eye, OS left eye 
uncontrolled uveitis and increased IOP, a pars plana vitrectomy was done. Thereafter, the uveitis resolved and the IOP became normal. OCT showed a CMT of 373 after vitrectomy and about 2 months after adalimumab injection (Fig. 1).

\section{Discussion}

This study explored the potential for adalimumab to be added to the armamentarium against post-cataract surgery inflammation. Given past evidence for efficacy of the mouse-originated infliximab, it was hypothesized that the humanized TNF- $\alpha$ inhibitor agent adalimumab may have beneficial effects with greater safety. [19-21]. Adalimumab is the most recent anti-TNF- $\alpha$ introduced and approved by the United States. This drug has been widely studied for the treatment of uveitis [17]. Although promising results were achieved on the anti-inflammatory effect of adalimumab in uveitis [17] little is known about the effect of adalimumab in cataract induced CME. The present study was designed to evaluate the effect of adalimumab in patients with pseudophakic macular edema. In our small patient cohort, beneficial outcomes were not achieved. There are several reports on the safety and efficacy of adalimumab in animal and experimental models. In rabbit (vitreous volume $=1.5 \mathrm{ml}$ ), the intravitreal administration of $0.50 \mathrm{mg}$ adalimumab is safe, but $1.0 \mathrm{mg}$ results in inflammation and retinal necrosis [19]. Androudi et al. [20] demonstrated that $1.0 \mathrm{mg}$ of adalimumab was not associated with adverse effects. Similarly, Manzano et al. [19] reported that the mean concentrations of adalimumab after the injection of $0.25,0.50$, and $1.0 \mathrm{mg}$ were $0.17,0.33$, and $0.67 \mathrm{mg} / \mathrm{ml}$, respectively, which are comparable to the injection of $0.75,1.5$, and $3.0 \mathrm{mg}$ adalimumab in the human eye according to the vitreous volume. Lihteh Wu et al. [16] demonstrated that intravitreal infliximab causes both anatomical and functional improvement in refractory pseudophakic CME, though no benefit in terms of vision gain and retinal thickness reduction were noted in our 5 patients with pseudophakic CME.

In another study, Lihteh Wu et al. [21] reported no benefit from intravitreal adalimumab and infliximab injections in patients with refractory diabetic macular edema. It seems that intravitreal injections of infliximab may lead to a severe intraocular inflammatory reaction. In the present study, one patient developed uveitis about 2 weeks after adalimumab injection which shows the risk of intravitreal injection of this drug.

Sofia Androudi et al. [20] reported no improvement in BCVA or central retinal thickness (CRT) reduction in patients with chronic uveitic macular edema treated with intravitreal adalimumab. In 2012, Farvardin et al. [22] reported BCVA improvement and decreased CMT in patients with chronic noninfectious uveitis treated with intravitreal infliximab, but its effect was temporary.

In the current study we also showed that adalimumab can lead to a transient decrease in CMT but there was no statistically significant difference in CMT values before injection, 1 week after injection, and 4 weeks after injection; nor was there any statistically significant difference in BCVA before injection, 1 week after injection, and 4 weeks after injection. Therefore, no beneficial effect for vision nor macular thickness was appreciated among our patients with pseudophakic macular edema treated with adalimumab. Additionally, inflammation-related safety concerns may also exist for this agent. Further study is needed to evaluate the efficacy and safety of intravitreal

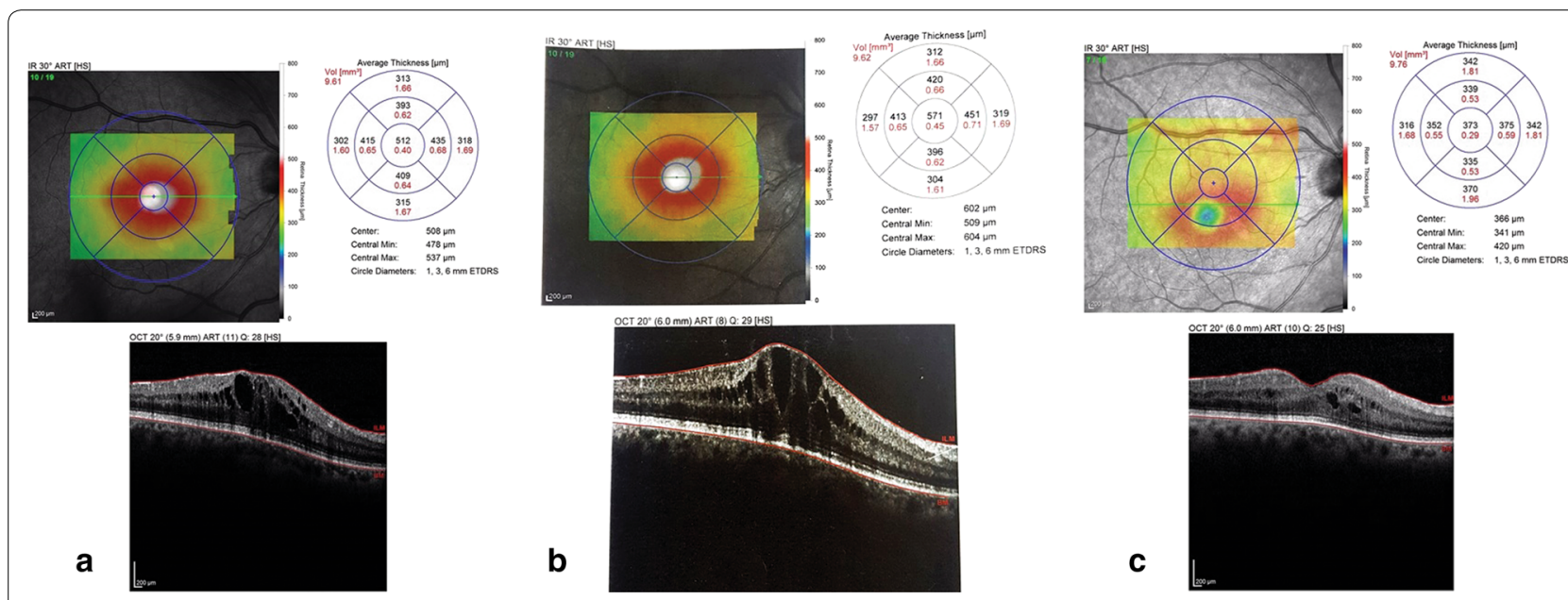

Fig. 1 OCT scans of the patient with sterile endophthalmitis (a) before injection, (b) 1 week after injection, (c) after vitrectomy. OCT represents CMT of 512 before injection, 5711 week after injection that decreased to 373 after vitrectomy 
adalimumab. According to the available data, this drug should be used with greater caution.

\section{Limitations}

Of the limitations of our study were the small sample size and the lack of a control group (including patients receiving intravitreal steroids). So, further study is suggested to evaluate the efficacy and safety of intravitreal adalimumab.

\begin{abstract}
Abbreviations
BCVA: Best corrected visual acuity; BRB: Blood-retinal barrier; CME: Cystoid macular edema; CMT: Central macular thickness; CRT: Central retinal thickness; IOP: Intra-ocular pressure; NSAIDs: Non-steroidal anti-inflammatory drugs; OCT: Optical coherence tomography; TNF-a:Tumor necrosis factor-alpha; VEGF: Vascular endothelial growth factor.
\end{abstract}

\section{Acknowledgements}

The authors would like to thank the directors of Shiraz University of Medical Sciences for supporting this research (Grant\# 6327).

\section{Authors' contributions}

M.F, E.N, and MK.J were involved in the conception and design of the study, acquisition of data, analysis, and interpretation of data, drafting the manuscript, and final revision. F.S-J was involved in drafting the manuscript, and final revision. All authors read and approved the final manuscript.

\section{Funding}

This study was supported by Shiraz University of Medical Science (Grant\# 6327).

\section{Availability of data and materials}

Not applicable.

\section{Ethics approval and consent to participate}

This study was approved by the ethics committee of Shiraz University of Medical Sciences (IR.SUS.REC.1394.99) and Iranian Registry of Clinical Trials (IRCT2016100430130N1) and informed written consents were obtained from patients.

\section{Consent for publication}

Not applicable.

\section{Competing interests}

The authors declare that they have no conflict of interest.

Received: 3 June 2020 Accepted: 18 July 2020

Published online: 25 July 2020

\section{References}

1. Flach AJ. The incidence, pathogenesis and treatment of cystoid macular edema following cataract surgery. Trans Am Ophthalmol Soc. 1998;96:557.

2. Gallego-Pinazo R, Arévalo JF, Udaondo P, García-Delpech S, Dolz-Marco R, Díaz-Llopis M. Prophylaxis of pseudophakic cystoid macular edema with intraoperative pegaptanib. J Ocul Pharmacol Ther. 2012;28(1):65-8.

3. Miyake K, Ibaraki N. Prostaglandins and cystoid macular edema. Surv Ophthalmol. 2002;47:S203-S218218.

4. Henderson BA, Kim JY, Ament CS, Ferrufino-Ponce ZK, Grabowska A, Cremers SL. Clinical pseudophakic cystoid macular edema: risk factors for development and duration after treatment. J Cataract Refract Surg. 2007;33(9):1550-8.

5. Tranos PG, Wickremasinghe SS, Stangos NT, Topouzis F, Tsinopoulos I, Pavesio CE. Macular edema. Surv Ophthalmol. 2004;49(5):470-90.

6. Conway MD, Canakis C, Livir-Rallatos C, Peyman GA. Intravitreal triamcinolone acetonide for refractory chronic pseudophakic cystoid macular edema. J Cataract Refract Surg. 2003;29(1):27-33.

7. Rho DS. Treatment of acute pseudophakic cystoid macular edema: diclofenac versus ketorolac. J Cataract Refract Surg. 2003;29(12):2378-84.

8. Steinert RF, Wasson PJ. Neodymium: YAG laser anterior vitreolysis for Irvine-Gass cystoid macular edema. J Cataract Refract Surg. 1989;15(3):304-7.

9. Harbour JW, Smiddy WE, Rubsamen PE, Murray TG, Davis JL, Flynn HW. Pars plana vitrectomy for chronic pseudophakic cystoid macular edema. Am J Ophthalmol. 1995;120(3):302-7.

10. Arevalo JF, Garcia-Amaris RA, Roca JA, Sanchez JG, Wu L, Berrocal $\mathrm{MH}$, et al. Primary intravitreal bevacizumab for the management of pseudophakic cystoid macular edema: pilot study of the PanAmerican Collaborative Retina Study Group. J Cataract Refract Surg. 2007;33(12):2098-105.

11. Arevalo JF, Maia M, Garcia-Amaris RA, Roca JA, Sanchez JG, Berrocal MH, et al. Intravitreal bevacizumab for refractory pseudophakic cystoid macular edema: the Pan-American Collaborative Retina Study Group results. Ophthalmology. 2009;116(8):1481-7.e1.

12. Rabinovich CE. Use of tumor necrosis factor inhibitors in uveitis. Curr Opin Rheumatol. 2007;19(5):482-6.

13. Santos Lacomba M, Marcos Martin C, Gallardo Galera J, Vidal MA, et al. Aqueous humor and serum tumor necrosis factor-a in clinical uveitis. Ophthalmic Res. 2001;33(5):251-5.

14. Muñoz-Fernández MA, Fresno M. The role of tumour necrosis factor, interleukin 6, interferon- $\gamma$ and inducible nitric oxide synthase in the development and pathology of the nervous system. Prog Neurobiol. 1998;56(3):307-40.

15. Khalili H, Lee RW, Khaw PT, Brocchini S, Dick AD, Copland DA. An anti-TNF-a antibody mimetic to treat ocular inflammation. Sci Rep. 2016:6:36905

16. Wu L, Arevalo JF, Hernandez-Bogantes E, Roca JA. Intravitreal infliximab for refractory pseudophakic cystoid macular edema: results of the Pan-American Collaborative Retina Study Group. Int Ophthalmol. 2012;32(3):235-43.

17. Neri P, Lettieri M, Fortuna C, Zucchi M, Manoni M, Celani S, et al. Adalimumab (Humira ${ }^{\text {TM }}$ ) in ophthalmology: a review of the literature. Middle East Afr J Ophthalmol. 2010;17(4):290.

18. Wiedmann MW, Mossner J, Baerwald C, Pierer M. TNF alpha inhibition as treatment modality for certain rheumatologic and gastrointestinal diseases. Endocr Metab Immune Disord Drug Targets. 2009;9(3):295-314.

19. Manzano RP, Peyman GA, Carvounis PE, Kivilcim M, Khan P, Chevez-Barrios $P$, et al. Ocular toxicity of intravitreous adalimumab (Humira) in the rabbit. Graefes Arch Clin Exp Ophthalmol. 2008;246(6):907-11.

20. Androudi S, Tsironi E, Kalogeropoulos C, Theodoridou A, Brazitikos P. Intravitreal adalimumab for refractory uveitis-related macular edema. Ophthalmology. 2010;117(8):1612-6.

21. Wu L, Hernandez-Bogantes E, Roca JA, Arevalo JF, Barraza K, Lasave AF. Intravitreal tumor necrosis factor inhibitors in the treatment of refractory diabetic macular edema: a pilot study from the Pan-American Collaborative Retina Study Group. Retina. 2011;31(2):298-303.

22. Farvardin M, Afarid M, Shahrzad S. Long-term effects of intravitreal infliximab for treatment of sight-threatening chronic noninfectious uveitis. J Ocul Pharmacol Ther. 2012;28(6):628-31.

\section{Publisher's Note}

Springer Nature remains neutral with regard to jurisdictional claims in published maps and institutional affiliations. 